\title{
REVISED Dynamics of Ebola epidemics in West Africa 2014
}

\section{[version 2; peer review: 2 approved]}

\section{Robin J. Evans', Musa Mammadov²}

1University of Melbourne, Parkville, 3010, Australia

${ }^{2}$ Federation University, Ballarat, 3350, Australia

V2 First published: 31 Dec 2014, 3:319

https://doi.org/10.12688/f1000research.5941.1

Latest published: 26 May 2015, 3:319

https://doi.org/10.12688/f1000research.5941.2

\section{Abstract}

This paper investigates the dynamics of Ebola virus transmission in West Africa during 2014. The reproduction numbers for the total period of epidemic and for different consequent time intervals are estimated based on a simple linear model. It contains one major parameter - the average infectious period that defines the dynamics of epidemics.

Numerical implementations are carried out on data collected from three countries Guinea, Sierra Leone and Liberia as well as the total data collected worldwide. Predictions are provided by considering different scenarios involving the average times of infectiousness for the next few months and the end of the current epidemic is estimated according to each scenario.

Keywords

simple linear model, dynamics, infectious period, infectiousness

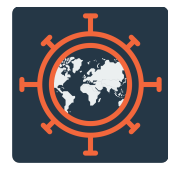

This article is included in the Emerging Diseases and Outbreaks gateway.

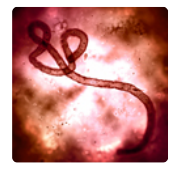

This article is included in the Ebola Virus

collection.

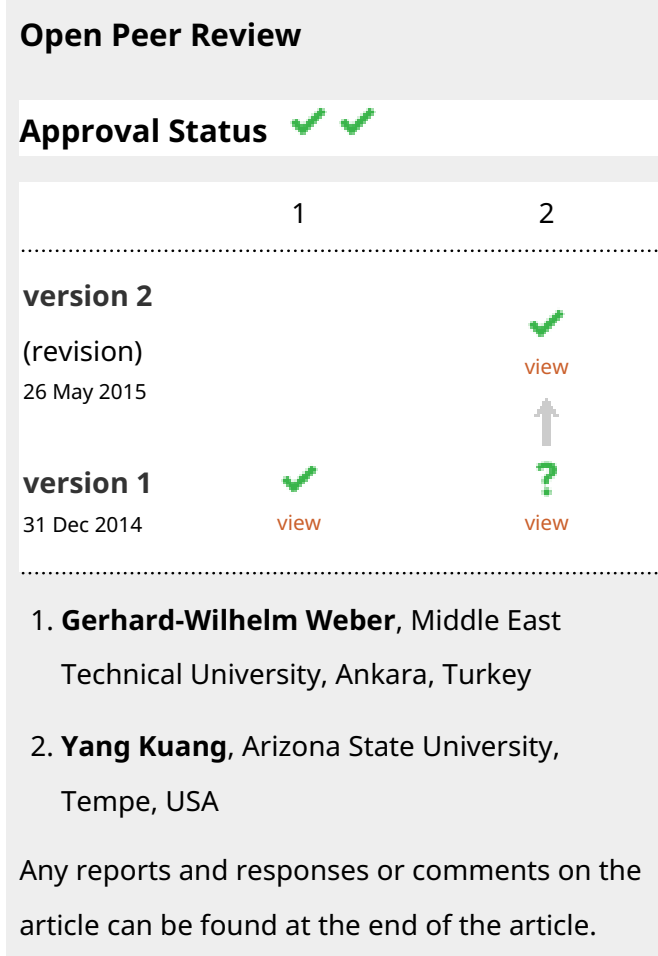


Corresponding author: Musa Mammadov (m.mammadov@federation.edu.au)

Competing interests: No competing interests were disclosed.

Grant information: This work was funded by the Collaborative Research Network (CRN) of the Federation University of Australia and the University of Melbourne.

Copyright: ( 2015 Evans RJ and Mammadov M. This is an open access article distributed under the terms of the Creative Commons Attribution License, which permits unrestricted use, distribution, and reproduction in any medium, provided the original work is properly cited.

How to cite this article: Evans RJ and Mammadov M. Dynamics of Ebola epidemics in West Africa 2014 [version 2; peer review: 2 approved] F1000Research 2015, 3:319 https://doi.org/10.12688/f1000research.5941.2

First published: 31 Dec 2014, 3:319 https://doi.org/10.12688/f1000research.5941.1 


\section{REVISED Amendments from Version 1}

This second version has two new references 5 and 10. Moreover in Section "Methods" the second paragraph in 'The effective reproduction numbers' subsection has been replaced by two new paragraphs

\section{See referee reports}

\section{Introduction}

The outbreak of the 2014 Ebola virus epidemic in West Africa, started in late 2013, does not seem to be under control and accurate predictions appear to be extremely difficult. The major reason for this might be due to unstable treatment conditions that provide different reproduction numbers at different periods. However, there are also other challenges related to the mathematical modeling of this epidemic. To address these challenges, several new models have been suggested that show quite different results, we note a few of them published recently ${ }^{1-8}$.

In this article we introduce a new model to study the dynamics of the current outbreak by considering the average infectious period as a time-dependent parameter. It is derived from the well studied SIR (Susceptible-Infectious-Recovery) model with time delay (e.g. 9,11), where the decrease in the number of susceptible population in compartment $S$ is the major force stopping epidemics. The susceptible population $S$ is often considered as a whole population. A major drawback of this model, in terms of the current epidemic, is that the population infected constitutes a very small proportion of the total population, a very small decrease in $S$ has almost no effect on compartment $I$.

We discuss how this drawback could be tackled and introduce a new model that uses only compartment $I$. This leads to a linear model having some similarities to those models based only on transmission rates from infectious population at different generations (e.g. 6). Our main goal is to fit data by estimating fewer and most influential parameters without considering many other issues like the infectiousness in hospitals and death ceremonies.

This in addition, allows us to have a more robust model with easily interpreted parameters that can be used for more accurate predictions. The main parameter in this model is the average infectious period $\tau_{2}$ (time from onset to hospitalization) that defines the dynamics of infectious population. This parameter can also be considered as a control parameter in the development of control models dealing with the spread of infection.

We calculate the basic reproduction numbers $\mathrm{R}_{0}$ for each country (Guinea, Sierra Leone and Liberia) as well as the total Ebola data worldwide. We also provide predictions corresponding to different scenarios by considering different values for $\tau_{2}$ for future time periods.

\section{Methods}

We use the notation $I_{a}(t)$ for the number of "active" infectious population at time $t$; it mainly represents the total number of infectious population that are not yet hospitalized. $C(t)$ and $D(t)$ are the cumulative number of infected cases and deaths, respectively. The population density of a country is denoted by $\mathscr{D}$. This is used in the definition of the infection force of the disease with coefficient $\beta$. Moreover, $\mu$ stands for the natural death rate of the population, $\alpha$ for the death rate due to disease, and $\tau_{1}$ for the average latent period (in days) that infected individuals become infectious and $\tau_{2}$ for the average infectious period (in days).

The main equations of our model are as follows (see Appendix for details):

$$
\begin{gathered}
I_{a}(t+1)=(1-\mu)^{\tau_{1}} \sum_{i=0}^{\tau_{2}-1}(1-\mu)^{i}(1-\alpha \omega(i)) \beta \mathscr{D} I_{a}\left(t-\tau_{1}-i\right) ; \\
C(t+1)=(1-\mu)^{\tau_{1}} \sum_{s=1}^{t} \beta \mathscr{D} I_{a}\left(s-\tau_{1}\right) ; \\
D(t+1)=(1-\mu)^{\tau_{1}} \sum_{s=1}^{t} \sum_{i=0}^{n}(1-\mu)^{i} \alpha \omega_{p}(i) \beta \mathscr{D} I_{a}\left(s-\tau_{1}-i\right) .
\end{gathered}
$$

Here $\omega$ is a gamma (cumulative) distribution function (with p.d.f $-\omega_{p}$ ) for deaths due to disease ${ }^{8}$; for the values of the parameters see Appendix. We note that there are only three parameters that need to be estimated to fit data for cumulative number of infected and death cases. These parameters are:

- $\quad \alpha$ - the death rate due to disease;

- $\quad \beta$ - the coefficient of the force of infection;

- $\tau_{2}$ - the average infectious period.

Here $\alpha$ and $\beta$ are continuous variables, $\tau_{2}$ is a discrete variable with integer values (days).

Basic Reproduction Number $-R_{0}$. We calculate the basic reproduction number by considering the stationary states in (1) as follows:

$$
R_{0}=\beta \mathscr{D}(1-\mu)^{\tau_{1}} \sum_{i=0}^{\tau_{2}-1}(1-\mu)^{i}(1-\alpha \omega(i)) .
$$


Since the natural death rate $\mu$ is close to zero; that is, $1-\mu \approx 1$, from (2) we have

$$
R_{0} \approx \beta \mathscr{D}\left[\tau_{2}-\alpha \sum_{i=0}^{\tau_{2}-1} \omega(i)\right]
$$

Moreover, since $\alpha \sum_{i=0}^{\tau_{2}-1} \omega(i)<1$, the reproduction number $R_{0} \approx \beta \mathscr{D} \tau_{2}$. This means that the reproduction number depends almost linearly on $\tau_{2}$.

The effective reproduction numbers $-R_{k}, k \geq 1$. The effective reproduction numbers $R_{k}$ are considered on several consecutive time intervals $\Delta_{k}=\left[t_{k}, t_{k+1}\right], k=1,2, \ldots$, with corresponding values of $\tau_{2}$. They are calculated by the same formula as $R_{0}$.

Here we make a reasonable assumption that the transmission rate $\beta \mathscr{D}$ describes the interaction of population (that is, in some sense, related to the local conditions and the life style) and should become relatively stable in the long term for a particular country. Then, the efforts in preventing the spread of infection are mainly observed in the change (decrease) in the value of $\tau_{2}$.

The infection rate is the key factor defining the dynamics of infectious population. The study ${ }^{5}$ shows that the infection rate is a linearly decreasing function of the total case reported. In 10 the 1995 Ebola outbreak in Congo is considered where the transmission coefficient $\beta$ is assumed to decrease exponentially due to control interventions. In our model the transmission of infection depends on two parameters $\beta$ and $\tau_{2}$. Here $\beta$ (having slightly a different meaning to what was used in 10) is constant and the dynamics of infectious populations depends on the change in $\tau_{2}$.

Therefore, to calculate the effective reproduction numbers, we fit data and find the optimal values for $\alpha$ and $\beta$, that are constant over the whole period, and optimal values $\tau_{2}^{\kappa}$ on each interval $\Delta_{k}$. Then $R_{k}$ is calculated by formula (2) setting $\tau_{2}=\tau_{2}^{k}$.

The sequence of optimal values $\tau_{2}^{1}, \tau_{2}^{2}, \ldots$, is considered as a method to describe the effectiveness of measures applied for preventing the spread of infection. This sequence very much defines the reproduction numbers on each consecutive time interval and therefore the dynamics of the infected population. It also allows us to consider future scenarios in terms of possible average infectious periods (i.e. times from onset to hospitalization).

\section{Results and discussion}

Data were retrieved from the WHO website (http://www.who. int/csr/disease/ebola/situationreports/en/) for the cumulative numbers of clinical cases (confirmed, probable and suspected) collected till 11 November 2014. In all numerical experiments, the second half of the available data for each country is used for fitting the cumulative numbers of infected cases and deaths. The global optimization algorithm DSO in Global And Non-Smooth Optimization (GANSO) library ${ }^{12,13}$ is applied for finding optimal values of parameters.

First we consider the whole period of infection in each country and find the best fit in terms of three variables $\alpha, \beta$ and $\tau_{2}$ (Problem $\left(D F_{1}\right)$ in Appendix). The results are presented in Table 1. Although from Figure 1 it can be observed that the best fit for Guinea is not as good as for the other cases, these results provide some estimate for the reproduction number $R_{0}$ for a whole period of infection till 11-Nov-2014. In all cases (except Guinea), $R_{0}$ is around 1.20 and for Guinea - 1.09. We note that the dynamics of infected population is much more complicated (especially in Guinea) which suggests that the reproduction number has been changing since the start of Ebola-2014 in almost all countries. This fact has been studied in 8 in terms of the instantaneous reproduction number over a 4-week sliding windows for each country (see also the next section for different values for $\tau_{2}$ ).

Table 1. Results of best fits: optimal values for
parameters $\alpha, \beta$ and $\tau_{2} . R_{0}$ is the reproduction number.

\begin{tabular}{|c|c|c|c|c|}
\hline Country & $\boldsymbol{\alpha}$ & $\boldsymbol{\beta}$ & $\boldsymbol{\tau}_{\mathbf{2}}$ (days) & $\boldsymbol{R}_{\mathbf{0}}$ \\
\hline Guinea & 0.632 & 0.00321 & 10 & 1.09 \\
\hline Sierra Leone & 0.371 & 0.00508 & 3 & 1.22 \\
\hline Liberia & 0.556 & 0.01090 & 3 & 1.17 \\
\hline World & 0.501 & 0.00362 & 7 & 1.21 \\
\hline
\end{tabular}

The effective reproduction numbers

According to (2), the basic reproduction number is mainly determined by $\beta$ and $\tau_{2}$. Since in our model parameter $\tau_{2}$ takes discrete values (days) it would be interesting to study the change of this parameter over time while keeping $\beta$ the same for the whole period. This approach makes it possible to consider different scenarios for future developments regarding the change in this parameter and to provide corresponding predictions.

We consider three consequent time intervals $\Delta_{k}=\left[t_{k}, t_{k+1}\right](k=1,2,3)$ for each country and find optimal values $\alpha, \beta$ and $\tau_{2}^{k}(k=1,2,3)$ (Problem $\left(\mathrm{DF}_{2}\right)$ in Appendix). The results are presented in Table 2. The last time point $t_{4}$ is 11-Nov-2014. The values of $t_{1}, t_{2}, t_{3}$ are as follows: 22-March, 23-May and 20-July for Guinea; 27-May, 20June and 20-August for Sierra Leone; 16-June, 20-July and 07-Sept for Liberia; and 22-March, 23-May and 07-Sept for the total data 
Guinea

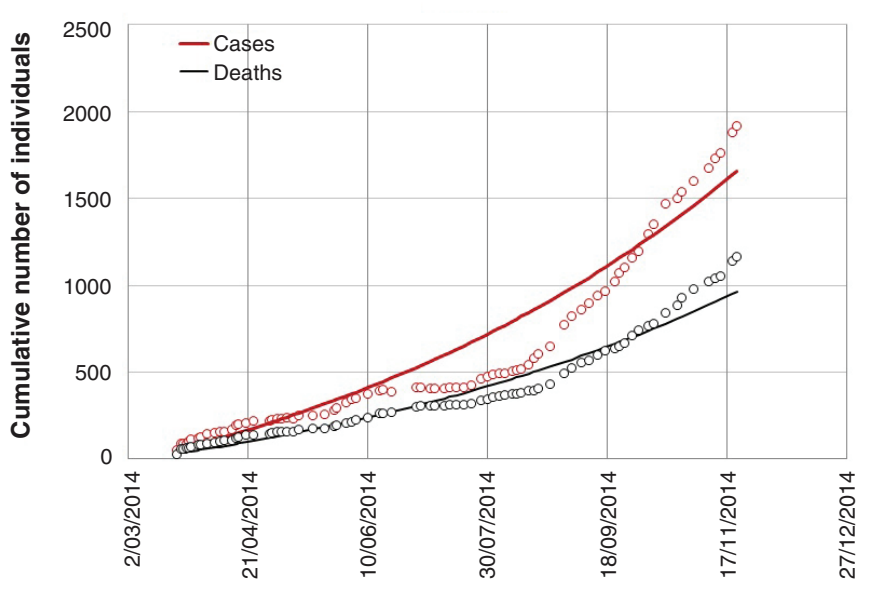

Liberia

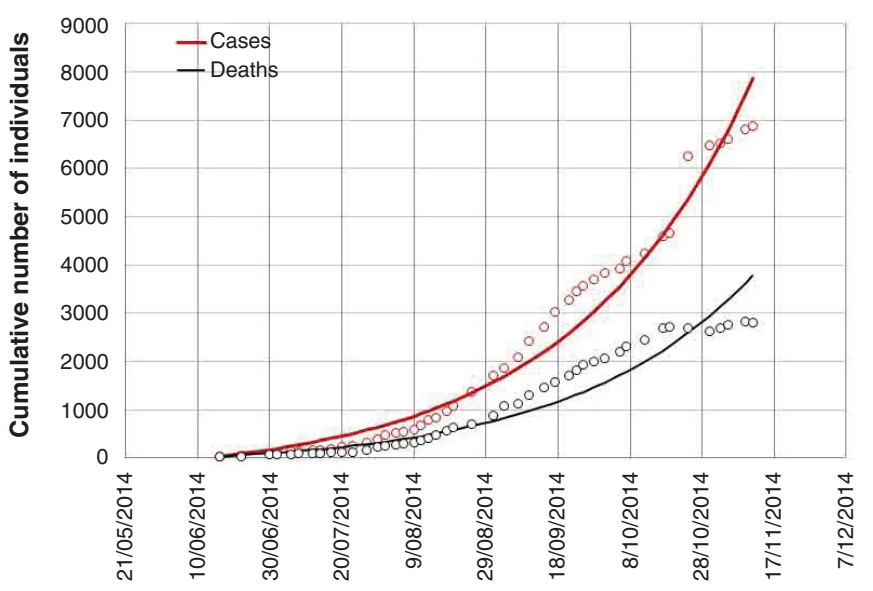

Sierra Leone

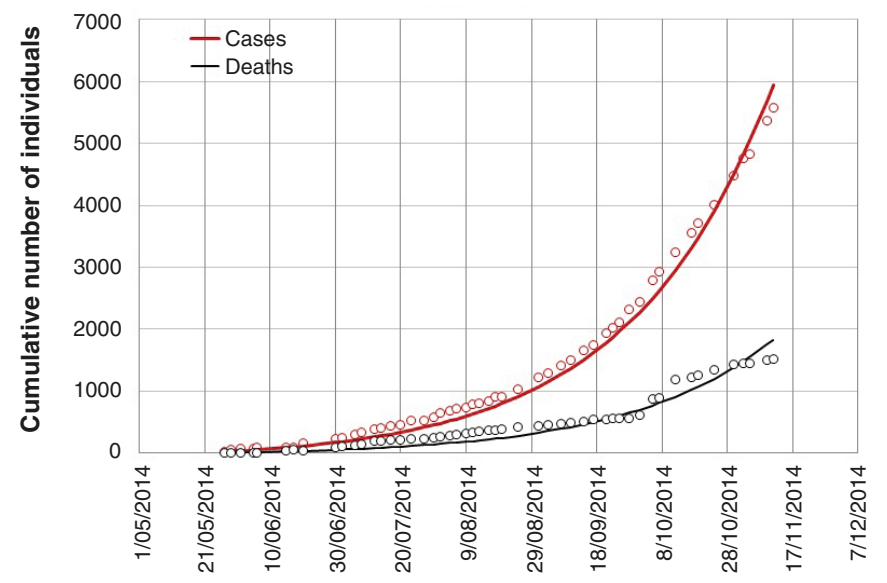

World

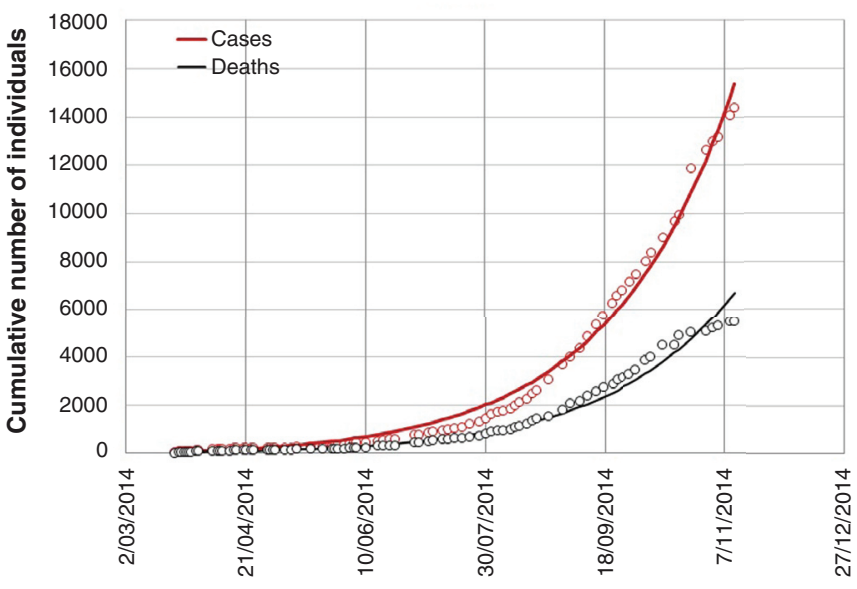

Figure 1. The best fits for the cumulative numbers of infected cases and deaths in Guinea, Sierra Leone, Liberia and worldwide by considering three parameters $\boldsymbol{\alpha}, \boldsymbol{\beta}$ and $\tau_{2}$ (for the values see Table 1). The lines represent the best fits, red and black circles represent the data.

Table 2. Results of best fits: the (effective) reproduction numbers $\boldsymbol{R}_{k}$ and average infectious period $\tau_{2}^{k}$ (in days) for different intervals $\Delta_{k}, \boldsymbol{k}=1,2,3$. The optimal values for $\alpha$ and $\beta$ are also provided; they are constant for a whole period.

\begin{tabular}{|c|c|c|c|c|c|}
\hline Country & $\boldsymbol{\alpha}$ & $\boldsymbol{\beta}$ & $\boldsymbol{R}_{\mathbf{1}}\left(\boldsymbol{\tau}_{\mathbf{2}}^{\mathbf{1}}\right)$ & $\boldsymbol{R}_{\mathbf{2}}\left(\boldsymbol{\tau}_{\mathbf{2}}^{\mathbf{2}}\right)$ & $\boldsymbol{R}_{\mathbf{3}}\left(\boldsymbol{\tau}_{\mathbf{2}}^{\mathbf{3}}\right)$ \\
\hline Guinea & 0.667 & 0.00527 & $0.86(4)$ & $1.25(6)$ & $1.07(5)$ \\
\hline Sierra L. & 0.353 & 0.00366 & $1.72(6)$ & $1.17(4)$ & $1.17(4)$ \\
\hline Liberia & 0.526 & 0.00688 & $1.45(6)$ & $1.23(5)$ & $0.99(4)$ \\
\hline World & 0.489 & 0.00519 & $1.04(4)$ & $1.29(5)$ & $1.04(4)$ \\
\hline
\end{tabular}


(World). Each interval $\Delta_{k}$ has its own reproduction number $R_{k}$ that defines the shape of the best fits presented in Figure 2.

In all cases the effective reproduction number is still greater than 1. In Liberia it shows a decrease from 1.45 to 0.99 and this can be seen in quite a noticeable decrease in the number of cumulative infected cases (Figure 2).

\section{Future scenarios}

We consider only the cumulative number of infected population worldwide. From Table 2 it can be observed that the number $\tau_{2}$ has changed as 4, 5 and 4 from 22-March to 11-Nov. We keep this initial best fit (the optimal values of parameters (World) are in Table 2) and consider different scenarios for possible changes of this parameter in the future while keeping the values of $\alpha$ and $\beta$ unchanged.

The future time intervals are designed as follows: the first interval $\Delta_{1}$ is $12 / \mathrm{Nov} / 2014-31 / \mathrm{Dec} / 2014$, followed by each next month $\Delta_{2}-\Delta_{4}$, and the last interval $\Delta_{5}$ starts from 1-Apr-2015. The results are presented in Table 3. The reproduction numbers are 0.778 (for $\tau_{2}=3$ ), 1.035 (for $\tau_{2}=4$ ) and 1.284 (for $\tau_{2}=5$ ).
Guinea

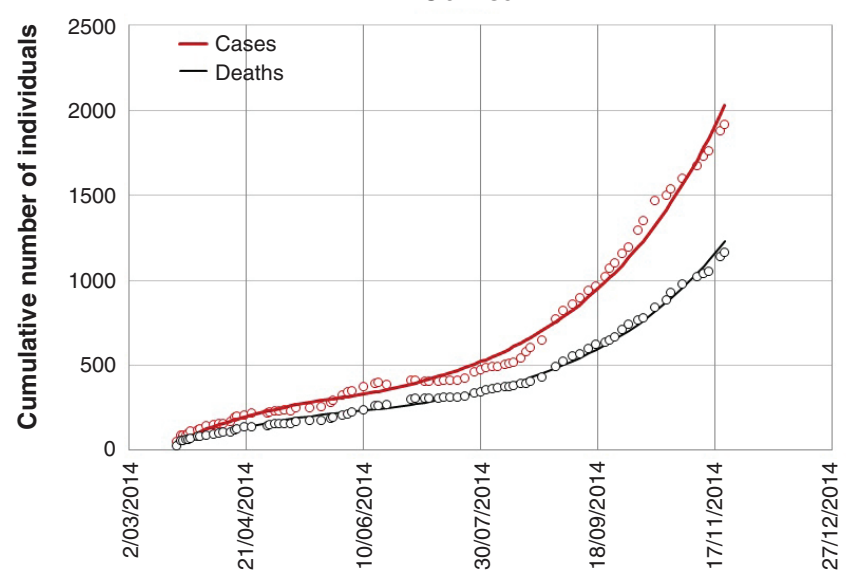

Liberia

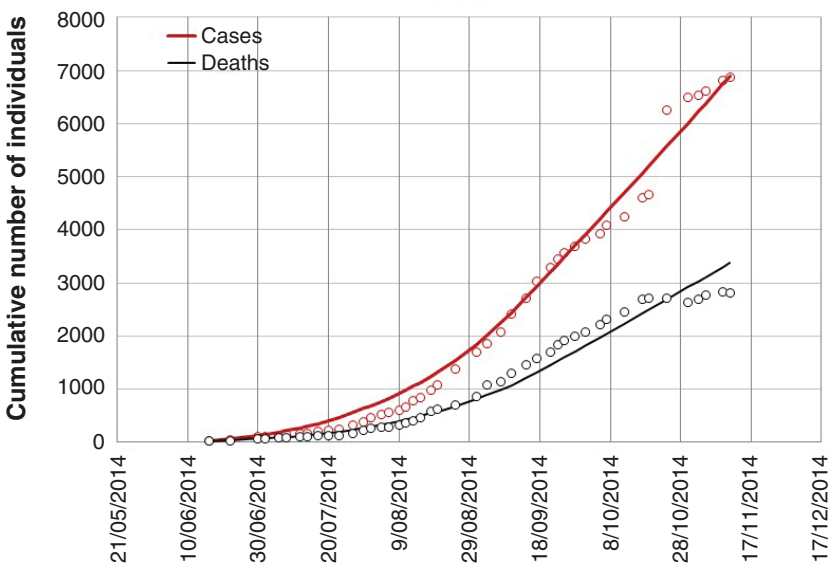

Sierra Leone

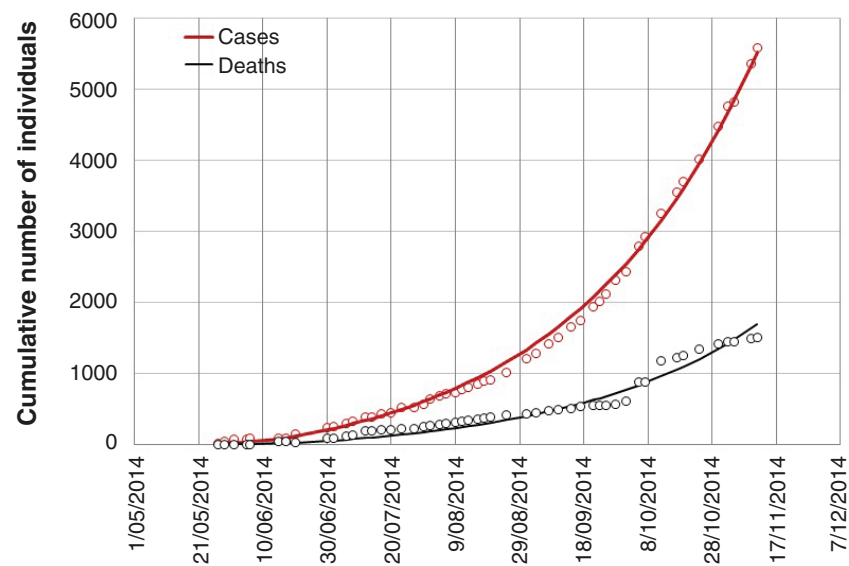

World

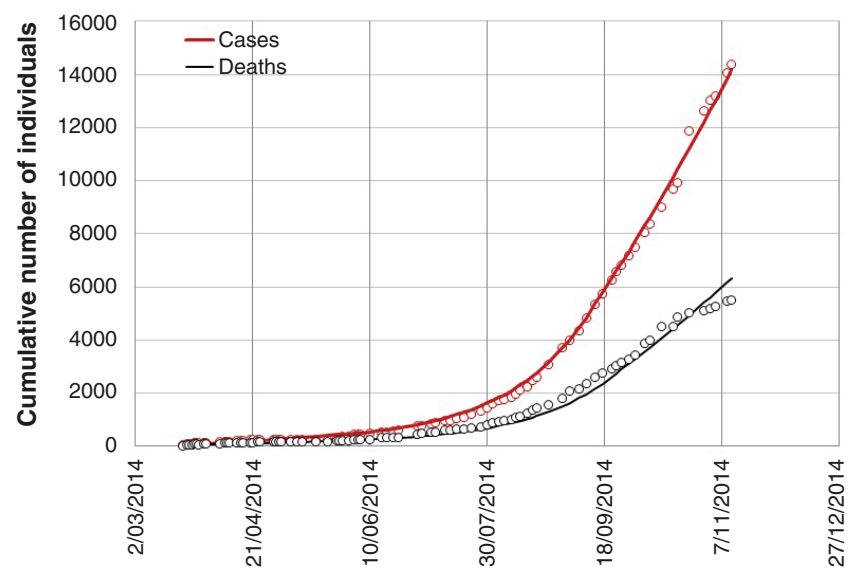

Figure 2. The best fits for the cumulative numbers of infected cases and deaths in Guinea, Sierra Leone, Liberia and worldwide by considering parameters $\alpha, \beta$ and three consequent time intervals with different values $\tau_{2}^{k}, k=1,2,3$ (for the values see Table 2). The lines represent the best fits, red and black circles represent the data. 
In the best scenario in Table 3 it is assumed that the current trend stays stable $\left(\tau_{2}=4\right)$ and a 25 percent decrease in the hospitalization time starts from 1-Jan-2015, then the epidemic may continue till Apr-2015 with the total number of infected cases reaching 31,000.

The worst case considered in Table 3 assumes that during the next two months (from 12-Nov-2014 to 31-Jan-2015) the average time to hospitalization increases by 25 percent (that is, from 4 days to 5 days) and then gradually decreases in Feb-Mar-2015 (from 5 days to 4 days), in Apr-2015 (from 4 days to 3 days) and stays at this level afterwards. In this case, the Ebola outbreak could be stopped by July-2015 with the total number of infected cases reaching 166,000 .

Table 3. The cumulative number of infected population according to different scenarios corresponding different values $\tau_{2}^{k+3}$ for time intervals $\Delta_{k^{*}}$. The starting values of parameters ( $\alpha$, $\beta$ and $\tau_{2}^{k}, k=1,2,3$ ) are in Table 2 (World). The first time interval $\left(\Delta_{1}\right)$ is $12 / \mathrm{Nov} / 2014-31 / \mathrm{Dec} / 2014$, followed by each next month and the last interval $\left(\Delta_{5}\right)$ starts from 1/Apr/2015. The last column presents the date for the end of Ebola epidemic (see also Figure 3) with corresponding number of cumulative infected population $C_{\text {max }}$ (-/ $\infty$ means no stabilization). The version $\tau_{2}^{k}=4$ for all $k$ means the current trend remains unchanged. The reproduction number for $\tau_{2}=3$ is $R=0.778$; it is less than 1 which leads to stabilization. For corresponding reproduction numbers for $\tau_{2}=4$ and 5 see Table 2 (World).

\begin{tabular}{|c|c|c|c|c|c|}
\hline $\boldsymbol{\tau}_{\mathbf{2}}^{\mathbf{4}}$ & $\boldsymbol{\tau}_{\mathbf{2}}^{\mathbf{5}}$ & $\boldsymbol{\tau}_{\mathbf{2}}^{\mathbf{6}}$ & $\boldsymbol{\tau}_{\mathbf{2}}^{\mathbf{7}}$ & $\boldsymbol{\tau}_{\mathbf{2}}^{\mathbf{8}}$ & End/C \\
\hline 4 & 4 & 4 & 4 & 4 & $-/ \infty$ \\
\hline 4 & 3 & 3 & 3 & 3 & Apr-2015/31,000 \\
\hline 4 & 4 & 3 & 3 & 3 & Apr-2015/39,000 \\
\hline 4 & 4 & 4 & 3 & 3 & May-2015/47,000 \\
\hline 4 & 4 & 4 & 4 & 3 & May-2015/57,000 \\
\hline 4 & 5 & 4 & 3 & 3 & Jun-2015/69,000 \\
\hline 4 & 5 & 4 & 4 & 3 & Jun-2015/90,000 \\
\hline 4 & 5 & 5 & 4 & 3 & Jun-2015/135,000 \\
\hline 5 & 4 & 4 & 3 & 3 & Jun-2015/102,000 \\
\hline 5 & 5 & 4 & 3 & 3 & Jul-2015/120,000 \\
\hline 5 & 5 & 4 & 4 & 3 & Jul-2015/166,000 \\
\hline
\end{tabular}

Future Scenarios

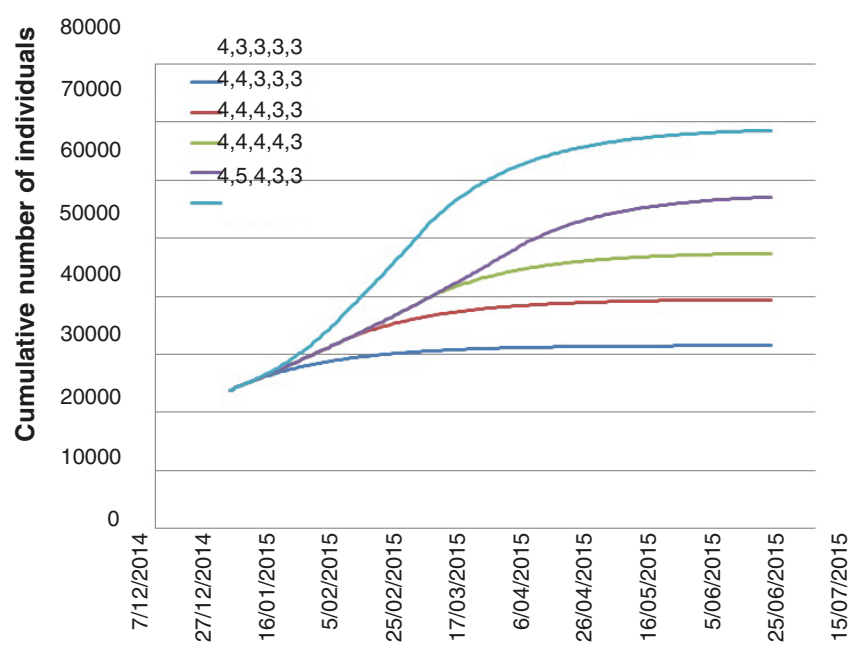

Future Scenarios

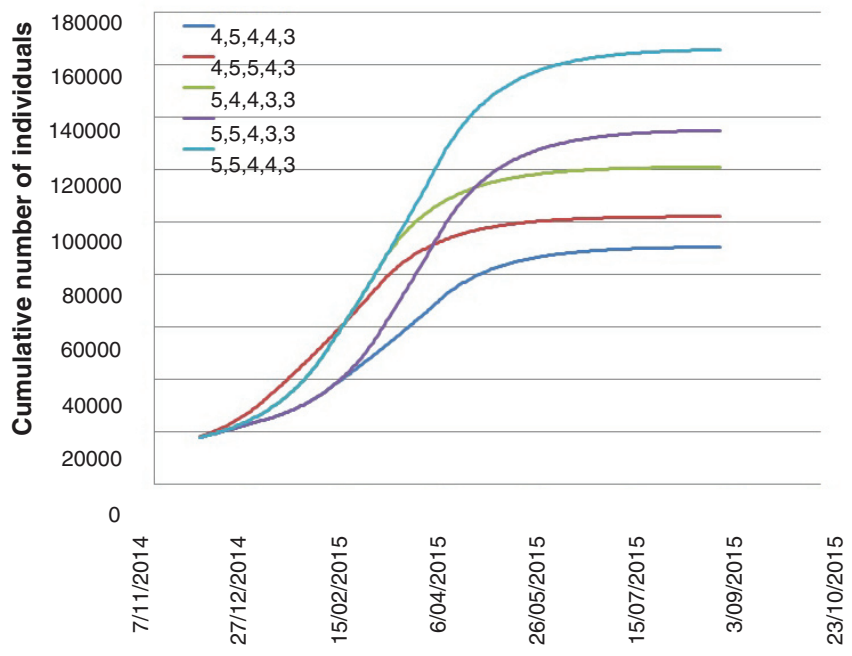

Figure 3. The cumulative number of infected population according to different scenarios corresponding different values $\tau^{k+3}$ for time intervals $\Delta_{k^{*}}$. The starting values of parameters $(\alpha, \beta$ and $\left.\tau_{2}^{k}, k=1,2,3\right)$ are in Table 2 (World). The first time interval $\left(\Delta_{1}\right)$ is $12 / \mathrm{Nov} / 2014-31 / \mathrm{Dec} / 2014$, followed by each next month and the last interval $\left(\Delta_{5}\right)$ starts from 1/Apr/2015. The reproduction number for $\tau_{2}=3$ is $R=0.778$; it is less than 1 which leads to stabilization. For corresponding reproduction numbers for $\tau_{2}=4$ and 5 see Table 2 (World). 


\section{Author contributions}

All authors contributed equally to the writing and subsequent refinement of the manuscript.

\section{Competing interests}

No competing interests were disclosed.

\section{Grant information}

This work was funded by the Collaborative Research Network $(\mathrm{CRN})$ of the Federation University of Australia and the University of Melbourne.

\section{Acknowledgements}

The first version of this paper is available in arXive.org: arXiv:1412.1579 [q-bio.PE].

\section{Appendix}

\section{Model}

The idea behind the model introduced in this paper is related to the SIR model with time delay. Since we are going to implement it on available daily data, a discrete version of this model is considered with the step-size one day. Moreover, since the "recovered" population is not our focus, we will only consider equations related to susceptible $(S)$ and infectious $(I)$ individuals. The most commonly used $S I R$ model $^{1}$ in the literature is provided below (see, for example, ${ }^{8}$ ):

$$
\begin{gathered}
S(t+1)=\lambda+(1-\mu) S(t)-F(S(t), I(t)) ; \\
I(t+1)=(1-\mu)^{\tau_{1}} F\left(S\left(t-\tau_{1}\right), I\left(t-\tau_{1}\right)\right)+(1-\mu-\alpha-\gamma) I(t) .
\end{gathered}
$$

Here $\lambda$ is the recruitment of the population; $\mu$ is the natural death rate of the population; $\alpha$ is the death rate due to disease; $\gamma$ is the recovery rate; and $\tau_{1}$ is the latent period that infected individual becomes infectious.

The fraction $(1-\mu)^{\tau_{1}}$ represents the survival rate of population over the period of $\left[0, \tau_{1}\right]$ (in continuous-time case it is equivalent to $e^{-\mu \tau_{1}}$ ). Below we examine this model in detail and develop an improved model.

Susceptible individuals. Equation (3) describes the dynamics of susceptible individuals $S(t)$. This equation "keeps" the number of infectious individuals $I(t)$ bounded. For example, when the basic reproduction number is greater than 1 , there exists ${ }^{8}$ an endemic equilibrium $\left(S^{*}, I^{*}\right)$ and $S(t) \rightarrow S^{*}, I(t) \rightarrow I^{*}$ as $t \rightarrow \infty$. In the case when the birth rate is zero $(\lambda=0)$ the relation $I(t) \rightarrow 0$ suggests that $S(t) \rightarrow 0$.

Thus according to this model the epidemic ends because the number of susceptible individuals $S(t)$ decreases over time and the effective reproduction number (as a function of time) becomes less than 1 at some stage; that is, the number of newly infected population $F(S(t)$, $I(t))$ decreases thanks to the "enough" decrease in the number of susceptible individuals (while $I(t)$ still increases). This might be applicable to epidemics in early 1900s but it is definitely not applicable to recent ones.
This issue significantly restricts the application of the SIR model for the study of the current Ebola virus epidemic. Below we consider 3 possibilities to overcome this difficulty.

1. The simplest way would be to use a "relatively small" number $S(0)$ for a possible number of susceptible individuals that may become infected. This approach has been implemented in ${ }^{1}$ where the total population size in each country (Guinea, Sierra Leone and Liberia) was assumed to be $10^{6}$ individuals.

2. An interesting (and most reliable in our opinion) approach would be considering "relatively small" number of population $S(0)$ as a variable that needs to be estimated. We have implemented this approach and the results show that currently available curve/data is not "long" enough to uniquely determine $S(0)$; that is, almost the same quality of data fit can be achieved for different numbers $S(0)$ (we have tried 50,000, 100,000 and 200,000) leading to different numbers of "stabilized" cumulative infected cases and infection periods. Taking this factor into account, we do not consider this approach, however we note that it might be quite possible soon with the availability of more data points.

3. In this paper we adopt another approach by neglecting the compartment $S$ completely and leaving just the compartment $I$. The force of infection $F(S, I)$ in this case is the main factor to be determined. We take this function in the form

$$
F(S, I)=\beta \mathscr{D} I
$$

where $\mathscr{D}$ is the population density of a particular country. In a more general setting, one would involve functions nonlinear in $I$ (like $F(S, I)=\beta \mathscr{D} I^{\xi}$ with $\left.\xi \leq 1\right)$. However, since the infectious population $I$ is a very small portion of the total population, function $F$ can be assumed linear at least in early stages of epidemics. In this case equation (4) can be represented in the form

$$
I(t+1)=(1-\mu)^{\tau_{1}} \beta \mathscr{D} I\left(t-\tau_{1}\right)+(1-\mu-\alpha-\gamma) I(t) .
$$

The major drawback of this model is that $I$ may growth infinitely if the reproduction number is greater than 1 ; in this model there is no variable/parameter (like $S(t)$ in $S I R$ ) that could force $I$ to decrease. On the other hand we believe that it can better describe the 
behavior of an infected population in "small" time intervals and provide more accurate reproduction numbers.

Active infectious population. Now we discuss the infectious population and equation (6) in more detail. We call "active infectious populations" at time $t$ the infected population that are infectious at that time but are not hospitalized yet. Denote by $I_{a}(t)$ the number of active infectious populations at time $t$. We will rewrite equation (6) in terms of $I_{a}$.

Denote by $\tau_{2}$ the average infectious period; that is, time from onset $\left(\tau_{1}\right)$ to hospitalization. Then, an infected person is assumed to be active infectious during the period $\left[\tau_{1}, \tau_{1}+\tau_{2}\right]$. Since $\tau_{2}$ is relatively small, we can assume that none is recovering during that period. This means that the rate of recovery $\gamma$ in (6) is no longer needed for $I_{a}(t)$.

Thus, we transform equation (6) by taking into account the time delay $\tau_{2}$. Accordingly, the equation for $I_{a}(t)$ can be represented in the form

$$
I_{a}(t+1)=(1-\mu)^{\tau_{1}} \sum_{i=0}^{\tau_{2}-1}(1-\mu)^{i}(1-\alpha \omega(i)) \beta \mathscr{D} I_{a}\left(t-\tau_{1}-i\right) .
$$

Here $\omega(0)=0$ and $\omega(i), i \geq 1$, is a gamma cumulative distribution function for onset-to-death that well describes the current Ebola virus in West Africa ${ }^{7}$. We note that in this equation, for each $i \geq 1$, the fraction $(1-\alpha \omega(i))$ is applied to the remaining infectious $(1-\mu)^{i}$ $\beta \mathscr{D} I_{a}\left(t-\tau_{1}-i\right)$; that is, the death rate in (7) is slightly different from (6) (indeed, both $\mu$ and $\omega(i)$ are quite small and this leads to $1-\mu-$ $\alpha \omega(i) \approx(1-\mu)(1-\alpha \omega(i)))$.

Cumulative number of infected cases. The first term $(1-\mu)^{\tau} 1 \beta$ $\mathscr{D} I_{a}\left(t-\tau_{1}\right)$ in (7) describes the number of new cases at time $t$. The cumulative number of infectious cases at $(t+1)$ will be denoted by $C(t+1)$. It can be calculated as

$$
C(t+1)=(1-\mu)^{\tau_{1}} \sum_{s=1}^{t} \beta \mathscr{D} I_{a}\left(s-\tau_{1}\right)
$$

Cumulative number of deaths. To calculate the cumulative number of deaths at time $t$, we consider all infectious cases (hospitalized or not) in the interval $\left[t-\tau_{1}, t-n\right]$ where $n$ is a sufficiently large number. In particular we assume that death may occur after the onset. As mentioned above, the distribution of death is described by a gamma distribution function $\omega$ with its p.d.f - $\omega_{p}$. Then, the cumulative number of deaths due to disease can be calculated as

$$
D(t+1)=(1-\mu)^{\tau_{1}} \sum_{s=1}^{t} \sum_{i=0}^{n}(1-\mu)^{i} \alpha \omega_{p}(i) \beta \mathscr{D} I_{a}\left(s-\tau_{1}-i\right)
$$

\section{Data fitting: Optimization Problems}

Main parameters. We have formulated the dynamical system (7),(8),(9). Given the observed cumulative number of infected cases - $C^{0}(t)$ and cumulative number of death cases - $D^{0}(t)$, the parameters of the systems can be estimated by the best fit. Before formulating this problem we discuss the parameters to be estimated.

The density $\mathscr{D}$ and the natural death rate of the population - $\mu$ is available for each country. We set $\mathscr{D}=41,80,36$ and 50 for Guinea, Sierra Leone, Liberia and the worldwide data, respectively. The natural death rate is around 10 deaths for 1000 population per year ( 1 percent yearly) for all the three countries. Thus in all numerical implementations, the daily rate $\mu$ is set to be $0.01 / 365=0.0000274$. It is reasonable to have the same average latency period - $\tau_{1}$ for infected individual to become infectious. The previous studies (e.g. 7) suggest that it is between 2-21 days with the mean of 11.4 days. Our numerical experiments show that the values between $6-8$ provide better results; we set $\tau_{1}=6$ in all cases.

Parameters of the gamma distribution can be taken from 7 . We set

$$
\omega_{p}(x)=\frac{b^{a}}{\Gamma(a)} x^{a-1} e^{-b x}, a=10, b=1.3333(10)
$$

with mean value 7.5. Note that the choice of values $a$ and $b$ within reasonable intervals, by keeping the mean value the same, has almost no effect on the quality of data fitting. Taking into account this fact, the parameters of the gamma distribution are chosen as in (10). In all the calculations, we set $n=35$ (days) in (9) (note that for large $i$ function values $\omega(i)$ are almost zero).

Initial values $I_{a}(t), t \leq 1$, for the equation are chosen in the form

$$
I_{a}(t)=\xi C^{0}(1), \text { for all } t \leq 1
$$

where $C^{0}(1)$ is the actual cumulative infectious. Numerical experiments show that the choice of $\xi$ in the interval $0.4-0.7$ has very little impact on the quality of data fitting. We set $\xi=0.4$ in all cases except Liberia for which the value 0.7 was better. Accordingly, we do not consider $\xi$ as a variable and set the above mentioned values for each country/data.

Therefore, the main parameters that define the dynamics of Ebola epidemics in different countries are $\alpha$ - the death rate due to disease, $\beta$ - the coefficient of the force of infection and $\tau_{2}$ - the average infectious period.

Data fitting. We consider data collected till 11 November 2014 for the cumulative number of infectious (confirmed, probable and suspected) and death individuals; they will be denoted by $C^{0}(t)$ and $D^{0}(t)$, 
respectively. We will use the root mean square error. Given time interval $\left[T_{1}, T_{2}\right]$ and data points $C^{0}\left(t_{i}\right)$ and $D^{0}\left(t_{i}\right), i \geq 1$, we define

$$
O\left(\left[T_{1}, T_{2}\right]\right)=\sum_{t_{i} \in\left[\frac{T_{2}-T_{1}}{2}, T_{2}\right]}\left[\left(C\left(t_{i}\right)-C^{0}\left(t_{i}\right)\right)^{2}+\left(D\left(t_{i}\right)-D^{0}\left(t_{i}\right)\right)^{2}\right] .
$$

According to this formula, we fit the second half of given data in order to decrease the choice of initial values $I_{a}(t), t \leq 1$, defined by (11).

Basic reproduction number $R_{0}$

To calculate the basic reproduction number, the above model is considered on the whole interval. The corresponding data fitting problem is:

Problem $\left(D F_{1}\right)$ : Given data $C^{0}\left(t_{i}\right)$ and $D^{0}\left(t_{i}\right), i \geq 1$, and time interval $\left[1, T_{2}\right]$ :

$$
\text { Minimize } f\left(\alpha, \beta, \tau_{2}\right)=\sqrt{O\left(\left[1, T_{2}\right]\right)}
$$$$
\text { subject to (7) }-(11) \text {. }
$$

The reproduction numbers $R_{k}, k=1,2,3$ for different time sections

The reproduction number is mainly determined by $\beta$ and $\tau_{2}$. Since in our model parameter $\tau_{2}$ takes discrete values (days) it would be interesting to study the change of this parameter over time while keeping $\beta$ the same for a whole period.

We consider three consequent time intervals $\Delta_{k}=\left[t_{k}, t_{k+1}\right](k=1,2,3)$ for each country and find optimal values $\alpha, \beta$ and $\tau_{2}^{k}(k=1,2,3)$. The last time point $t_{4}$ is $T_{2}=11-N o v-2014$. Corresponding data fitting problem is

Problem $\left(D F_{2}\right)$ : Given data $C^{0}\left(t_{i}\right)$ and $D^{0}\left(t_{i}\right), i \geq 1$, and time interval $\left[t_{1}, t_{4}\right]$ :

$$
\text { Minimize } f\left(\alpha, \beta, \tau_{2}^{1}, \tau_{2}^{2}, \tau_{2}^{3}\right)=\sqrt{O\left(\left[t_{1}, t_{4}\right]\right)}
$$
subject to: $(7)-(11)$, where in (7)

$$
\tau_{2}=\tau_{2}^{k}, \forall t \in \Delta_{k}, k=1,2,3
$$

${ }^{1}$ Continuous time version of this model is

$$
\begin{gathered}
\frac{d S(t)}{d t}=\lambda-\mu S(t)-F(S(t), I(t)), \\
\frac{d I(t)}{d t}=e^{-\mu \tau_{1}} F\left(S\left(t-\tau_{1}\right), I\left(t-\tau_{1}\right)\right)-(\mu+\alpha+\gamma) I(t)
\end{gathered}
$$

1. Althaus $C L$ : Estimating the reproduction number of ebola virus (EBOV) during the 2014 outbreak in West Africa. PLoS Currents Outbreaks. 2014. Publisher Full Text

2. Browne C, Huo X, Magal P, et al:: A model of the $\mathbf{2 0 1 4}$ Ebola epidemic in West Africa with contact tracing. arXiv preprint arXiv:1410.3817. 2014. Reference Source

3. Chowell G, Hengartner NW, Castillo-Chavez C, et al.: The basic reproductive number of Ebola and the effects of public health measures: the cases of Congo and Uganda. J Theor Biol. 2004; 229(1): 119-126. PubMed Abstract | Publisher Full Text

4. Chowell G, Nishiura $\mathrm{H}$ : Transmission dynamics and control of Ebola virus disease (EVDI): a review. BMC Med. 2014; 12(1): 196. PubMed Abstract | Publisher Full Text | Free Full Text

5. Chowell G, Simonsen L, Viboud C, et al.: Is West Africa Approaching a Catastrophic Phase or is the 2014 Ebola Epidemic Slowing Down? Different Models Yield Different Answers for Liberia. PLOS Curr. 2014; Edition 1. PubMed Abstract | Publisher Full Text | Free Full Text

6. Nishiura H, Chowell G: Early transmission dynamics of Ebola virus disease (EVD), West Africa, March to August 2014. Euro Surveill. 2014; 19(36). pii: 20894. PubMed Abstract

7. Rivers $\mathrm{CM}$, Lofgren $\mathrm{ET}$, Marathe M, et al.: Modeling the impact of interventions on an epidemic of Ebola in Sierra Leone and Liberia. arXiv preprint
arXiv:1409.4607. 2014

Reference Source

8. WHO Ebola Response Team. Ebola virus disease in West Africa--the first 9 months of the epidemic and forward projections. N Engl J Med. 2014; 371(16): 1481-95. PubMed Abstract | Publisher Full Text | Free Full Text

9. Li M, Liu X: An SIR epidemic model with time delay and general nonlinear incidence rate. $2014 ; 2014: 1-7$.

Publisher Full Text

10. Lekone PE, Finkensatdt BF: Statistical inference in a stochastic epidemic SEIR model with control intervention: Ebola as a case study. Biometrics. 2006; 62(4): 1170-1177.

PubMed Abstract | Publisher Full Text

11. Wang J, Wang J, Liu M, et al.: Global stability analysis of an SIR epidemic model with demographics and time delay on networks Physica A: Statistical Mechanics and its Applications. 2014; 410: 268-275. Publisher Full Text

12. Global and Non-Smooth Optimization library (GANSO). Federation university Australia. Reference Source

13. Mammadov M, Rubinov A, Yearwood J: Dynamical systems described by relational elasticities with applications. Continuous Optimisation: Current Trends and Modern Applications, V. Jeyakumar and A. Rubinov (Eds). 2005; 99: 365-385. Publisher Full Text 


\section{Open Peer Review}

\section{Current Peer Review Status:}

\section{Version 2}

Reviewer Report 27 May 2015

https://doi.org/10.5256/f1000research.7037.r8759

(C) 2015 Kuang Y. This is an open access peer review report distributed under the terms of the Creative Commons Attribution License, which permits unrestricted use, distribution, and reproduction in any medium, provided the original work is properly cited.

\section{Yang Kuang}

School of Mathematical and Statistical Sciences, Arizona State University, Tempe, AZ, USA

The authors adequately responded to my comment on the assumption that the transmission is constant.

Competing Interests: No competing interests were disclosed.

I confirm that I have read this submission and believe that I have an appropriate level of expertise to confirm that it is of an acceptable scientific standard.

\section{Version 1}

Reviewer Report 01 May 2015

\section{https://doi.org/10.5256/f1000research.6350.r8331}

(C) 2015 Kuang Y. This is an open access peer review report distributed under the terms of the Creative Commons Attribution License, which permits unrestricted use, distribution, and reproduction in any medium, provided the original work is properly cited.

\section{Yang Kuang}

School of Mathematical and Statistical Sciences, Arizona State University, Tempe, AZ, USA

This manuscript introduced some novel ways to connect the current West Africa Ebola outbreak to the standard SI model. However, the author routinely assumed that the infection rate $\beta$ is a constant which more or less causes the exponential growth profile even when indications suggest otherwise after the month of October, 2014. The key to better modeling any infectious disease and especially in severe ones like Ebola, is to appropriately model the infection rate which is really 
a function of individual behavior. Arguably, individual behavior is often strongly correlated to the total cases reported. I suggest that the authors take a look at the paper by Chowell et al. below which implicitly incorporated such observations. One can show by a direct differentiation of the logistic model in the paper by Chowell et al. to find out that I'/I is assumed to be a linearly decreasing function of the total case reported.

Chowell G, Simonsen L, Viboud C, Kuang Y. 2014. Is West Africa approaching a catastrophic phase or is the 2014 Ebola epidemic slowing down? Different models yield different answers for Liberia. PLOS Currents Outbreaks. 2014 Nov 20. Edition 1. doi: 10.1371/currents.outbreaks. b4690859d91684da963dc40e00f3da81. PLOS Currents Outbreaks.

Competing Interests: No competing interests were disclosed.

\section{I confirm that I have read this submission and believe that I have an appropriate level of expertise to confirm that it is of an acceptable scientific standard, however I have significant reservations, as outlined above.}

Author Response 13 May 2015

Musa Mammadov, Federation University, Ballarat, Australia

We thank the reviewer for pointing out the importance of the infection rate which is really a key focus of our paper. Our model mainly depends on two parameters: $\beta$ and $\tau_{2}-$ time to isolation. Initially we also thought that $\beta$ should be time dependent (in fact piece-wise constant) and obtained good data fitting in this way. However, later we observed that almost the same quality of data fitting can be achieved if we take $\beta$ constant and vary only $\tau$ 2. This is one the major findings of our paper and has important implications. For example:

Since $\tau_{2}$ is an integer it is possible to generate future scenarios for the prediction of infectious population.

As $\tau_{2}$ is time to isolation it can be linked with time to hospitalization and can lead to optimal control problems.

Such an optimal control model has already been implemented in our new study ( arXiv:1505.00872) where the problem of optimal distribution of bed capacities is investigated.

The paper by "Chowell et al" is cited in a new version regarding the behavior of $\beta$, alongside "Lekone et al" where $\beta$ is considered as a control variable with an exponential decrease.

Competing Interests: No competing interests

Reviewer Report 12 March 2015

\section{https://doi.org/10.5256/f1000research.6350.r7898}

(C) 2015 Weber G. This is an open access peer review report distributed under the terms of the Creative Commons Attribution License, which permits unrestricted use, distribution, and reproduction in any medium, provided the original work is properly cited. 


\section{Gerhard-Wilhelm Weber}

Institute of Applied Mathematics, Middle East Technical University, Ankara, Turkey

This is an excellent research work for which the researcher(s) related to the study deserve great thanks and a particular recognition!

The paper discloses beauty and rigor of modern Mathematics and Operational Research, and it has the potential to strongly contribute to health and entire living conditions of individuals, communities and nations.

Moreover, it can very much stimulate and initiate future research works and practical contributions, e.g., via stochastic aspects and regime switches or paradigm changes.

Competing Interests: No competing interests were disclosed.

I confirm that I have read this submission and believe that I have an appropriate level of expertise to confirm that it is of an acceptable scientific standard.

The benefits of publishing with F1000Research:

- Your article is published within days, with no editorial bias

- You can publish traditional articles, null/negative results, case reports, data notes and more

- The peer review process is transparent and collaborative

- Your article is indexed in PubMed after passing peer review

- Dedicated customer support at every stage

For pre-submission enquiries, contact research@f1000.com 